\title{
Comparison of Digital and Analog Silicon Photomultiplier For Positron Emission Tomography Application
}

\author{
Chen Xu, Erika Garutti, Shingo Mandai and Edoardo Charbon
}

\begin{abstract}
In recent years, silicon photomultipliers (SiPMs) became popular candidates for applications in positron emission tomography (PET) scanners or PET based multi-modality detectors. In this paper, we perform a comparative study of digital and analog SiPM in gamma spectroscopy with a lutetium-yttrium oxyorthosilicate (LYSO) scintillator. The energy spectrum in terms of photo-electron numbers (fired pixels) is obtained directly from the digital SiPM. The energy spectrum obtained with analog SiPM is converted to number of fired pixels using single photoelectron spectrum measurement. Non-linear behavior of both detectors is studied. Energy response for $511 \mathrm{keV}$ gamma ray under non-linear conditions for both detectors are corrected using a Monte-Carlo simulation tool.
\end{abstract}

\section{INTRODUCTION}

A Silicon photomultiplier is a matrix of identical single photon avalanche diodes (SPAD or pixel) operating in Geiger mode. Each pixel of a SiPM operates as an independent photon counter, i.e. a photon impinging on a pixel can create free carriers, which with a certain probability cause a Geiger discharge [1]. An analog SiPM (a-SiPM) sums the discharge current of all pixels on a common load resistor to form an analog output signal. The amplitude of the output signal is therefore proportional to the number of detected photons. A-SiPM coupled with inorganic scintillator makes a strong candidate for time-of-flight (TOF) positron emission tomography (PET) detectors [2]. Compared to photomultiplier tubes (PMT), SiPMs are more compact, have a lower operating voltage and are insensitive to magnetic field [3].

The digital SiPM (d-SiPM) approach integrates SPAD with conventional CMOS circuits on the same substrate [4], [5]. A one-bit memory can be used to selectively enable or disable the respective pixels which gives a handle on optimizing the overall dark counts. On chip Time-to-Digital Converters (TDC) provide timing information with minimum noise. As a result, an extreme miniaturized PET detector may be achievable with the d-SiPM architecture.

The EndoTOFPET-US project [6] proposes an asymmetric TOFPET detector joint with ultrasound endoscopic probe as a multi-modality instrument for the development of new biomarkers for pancreas and prostate oncology. The internal PET probe design foresees a custom developed digital SiPM

C. Xu is with DESY, D-22761 Hamburg, Germany.

E. Garutti is with the Institute of Experimental Physics, University of Hamburg, Luruper Chaussee 149, D-22761 Hamburg, Germany,

S. Mandai and E. Charbon are with the Faculty of Electrical Engineering, Delft University of Technology, Mekelweg 4, 2628, CD Delft, The Netherlands, with multiple integrated TDCs called multi-channel digital SiPM (MD-SiPM) [5], [7]. A prototype of the MD-SiPM is developed by collaboration members from Delft University of Technology. A single MD-SiPM is divided into 416 pixels, has a detector area of $800 \times 780 \mu \mathrm{m}^{2}$ and up to $17 \%$ photodetection efficiency (PDE) at $4 \mathrm{~V}$ excess bias [5]. In this paper, we present the performance of the prototype sensor coupled to a $1 \times 1 \mathrm{~mm}^{2}$ LYSO scintillating crystal. The photon energy spectrum of ${ }^{22} \mathrm{Na}$ source is presented in order to demonstrate the functionality of the prototype sensor. As a comparison, a $1 \times 1 \mathrm{~mm}^{2}$ Multi-Pixel-Photon-Counter (MPPC) from Hamamatsu (type S10362- 11-050C) [8] with $50 \mu \mathrm{m}$ pixel pitch and 400 pixels is used to read out the same crystal. Due to limited number of pixels in both sensors, the response to the scintillating light from a crystal is strongly non-linear. A Monte-Carlo software is developed to simulate the behaviors of MD-SiPM and MPPC for the non-linearity correction of the sensors.

\section{MD-SiPM PROTOTYPE}

\section{A. Architecture}

In this work, a $800 \times 780 \mu \mathrm{m}^{2}$ prototype MD-SiPM with 416 pixels is tested. Details of the chip design can be found in [7], specifications relevant to the energy measurement are described here. The single pixel size is $50 \times 30 \mu \mathrm{m}^{2}$ with a fill factor of 57\%. The photo-detection efficiency (PDE) of the sensor operating at $2.5 \mathrm{~V}$ excess bias is $12.5 \%$. Each pixel consists of a SPAD with a 1-bit counter for pixel firing registration and circuits for pixel masking and signal shaping. The signal from a pixel is routed directly to one of the 3 TDCs shared by a column of pixels in an interlaced configuration. In total 48 TDCs are available for the sensor.

The chip operates in self-triggered mode with framed readout. The start signal pulse sent to the device clears all pixel counters and starts the TDC clock. A firing SPAD due to photon detection or dark count, causes the 1-bit counter's increment, and stops the connected TDC. Therefore the pixel firing time is recorded. The 1-bit counter records only the first firing of a pixel and therefore eliminates after pulses. At the end of the frame, the counted number of fired pixels and TDC data are read out. The frame acquisition time can be defined by the user before starting a measurement.

\section{B. Dark Count Rate}

One characteristics of a MD-SiPM is its Dark Count Rate (DCR) in count per second (cps) as function of excess bias 

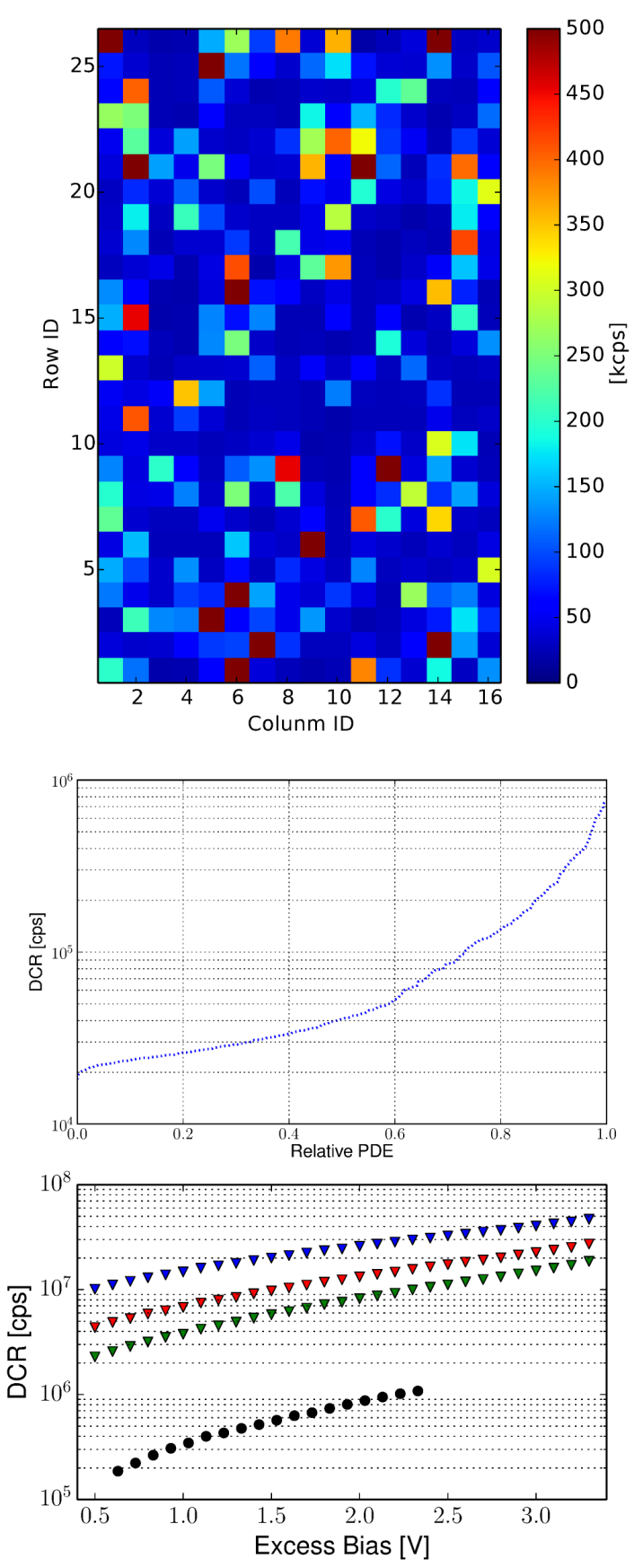

Fig. 1. Dark count rate of a MD-SiPM at $20^{\circ} \mathrm{C}, 2.5 \mathrm{~V}$ excess voltage and its comparison to a MPPC. The top image shows the pixel-by-pixel dark count rate map of the sensor. The $\mathrm{z}$ axis is given in kilo-counts per second ( $\mathrm{kcps}$ ). The map demonstrates that noisy pixels are randomly distributed over the MD-SiPM. The middle plot shows the total DCR as a function of "relative PDE" as defined in the text. The bottom plot shows the dependence of DCR on excess bias voltage for a MD-SiPM and a MPPC.

above the break down voltage. DCR is measured on a pixelby-pixel basis for the MD-SiPM (shown in Fig. 1 top), and the overall DCR of the device is the sum of all pixels' DCR. The measurement is performed in a light-tight environment. Assuming the random occurrence of a dark count follows Poisson statistic, the probability $\left(P_{0}\right)$ of not observing a dark
TABLE I

DCR PER UNIT DETECTER AREA OF A MPPC AND A MD-SIPM

\begin{tabular}{|c|c|c|}
\hline & MPPC & MD-SiPM \\
\hline DCR $\left[\mathrm{cps} / \mu \mathrm{m}^{2}\right]$ & 0.5 & 50 \\
\hline
\end{tabular}

event in the frame acquisition time, $\Delta t$, is given by:

$$
P_{0}=e^{-D C R \cdot \Delta t} \rightarrow D C R=-\frac{\ln \left(P_{0}\right)}{\Delta t}
$$

where $P_{0}$ is the ratio of frames without dark counts to the total number of frames. The frame acquisition time used in the DCR measurement is $100 \mathrm{~ns}$.

To reduce the total DCR of the MD-SiPM, pixels with high DCR of the MD-SiPM can be selectively turned off. However this also reduces the MD-SiPM's photo detection efficiency (PDE). The trend of trading off between DCR and PDE is shown in the middle plot of Fig. 1.

The bottom plot in Fig. 1 shows DCR's dependence on excess bias of the MD-SiPM with "relative PDE", the percentage of pixel masking. As a comparison, the DCR and its dependence on excess bias of MPPC is determined in the same fashion (DCR measurement for MPPC in section III-A) and shown in the same plot. Table I shows the DCR of both devices at their nominal operating voltage $(1.4 \mathrm{~V}$ excess bias for MPPC and $2.5 \mathrm{~V}$ excess bias for MD-SiPM) normalized to the unit detector area. All measurements are performed at $20^{\circ} \mathrm{C}$ using $100 \mathrm{~ns}$ time interval.

\section{Trigger Validation}

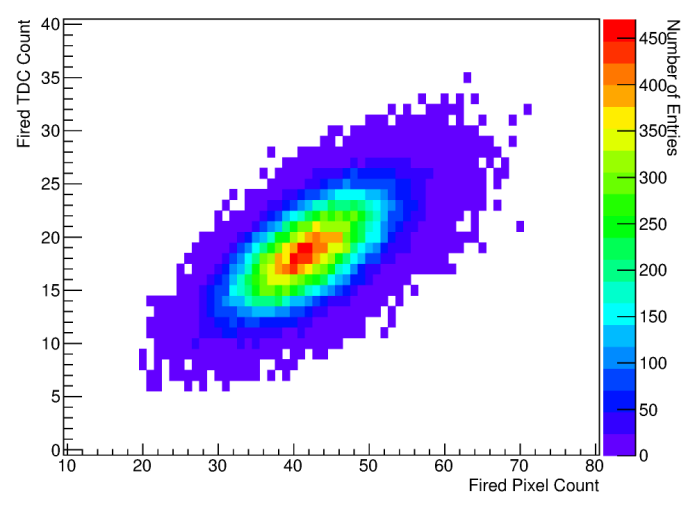

Fig. 2. Number of fired TDCs as a function of number of fired pixels in a frame of $400 \mathrm{~ns}$ without input light on MD-SiPM (only dark counts). Because of the shared TDC architecture, a mean number of 42 dark count fired pixels have triggered a mean number of 19 TDCs in a frame.

Operating in self-triggered mode with a fixed frame acquisition time, dark counts cause pixel firing and therefore reduce the dynamic range. They also introduce fake TDC activations whose time stamp is not the arrival time of a photon from the scintillation light. The occurrence of a scintillation event in PET application is random relative to the acquisition frame. Therefore a validation is required to periodically check the pixel and TDC activations and reset those who are introduced by dark counts. The "Smart Reset" (SR) function of the chip is implemented for this purpose. The 48 TDCs on the 


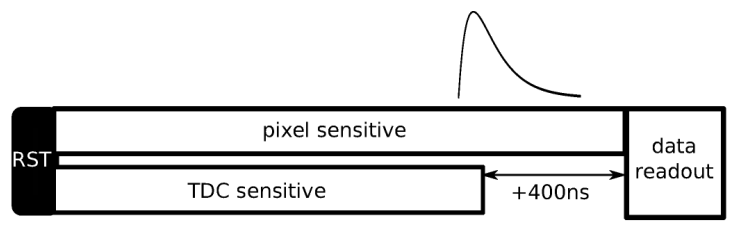

Fig. 3. The diagram shows the timing of pixels and the TDCs sensitive time in a frame acquisition time. When a scintillating event happens in the later part of a frame, multiple TDCs may have recorded the time of arrival of the early photons, an extended pixel working time of $400 \mathrm{~ns}$ allows the device to record the whole scintillating process.
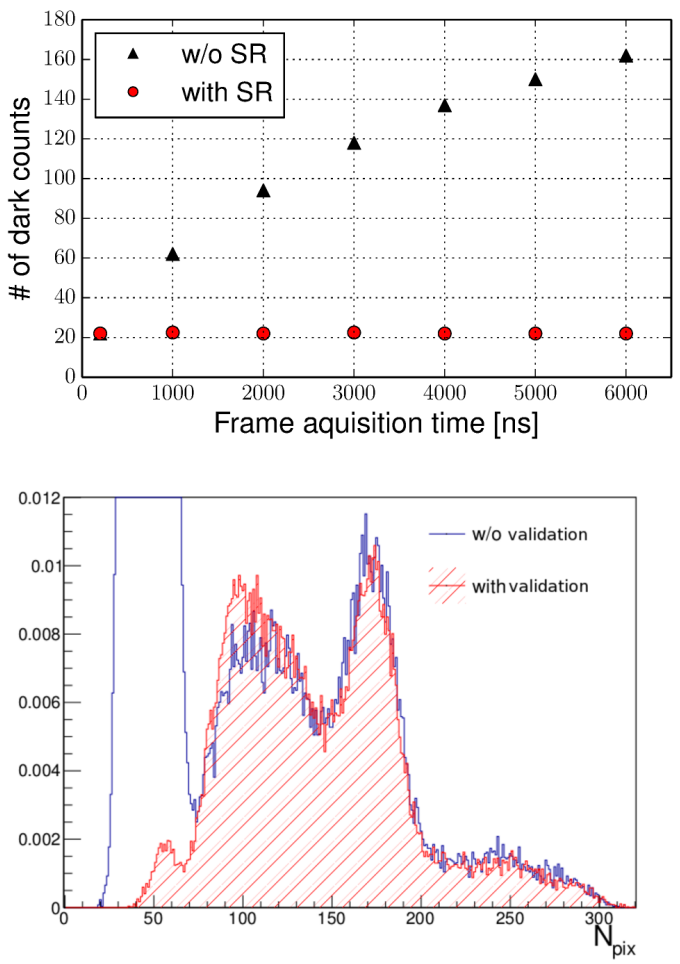

Fig. 4. Trigger validation functionality test. The top plot shows SR effectively keeps dark counts independent from frame acquisition time. The bottom plot shows spectra with and without trigger validation applied on the number of activated TDCs. A threshold of 30 is used. After applied validation, frames with valid scintillation events are effectively selected. The ratio between signal and noise events is improved.

chip give the device the capability of recording 48 timing information corresponding to the earliest arrived photons. For a low number of photons, the number of TDC measurements is proportional to the accumulated number of pixel fired (shown in Fig. 2). Due to the electronic scheme of the chip, it is faster to read out the number of activated TDCs without influencing the pixel operation. We expect the scintillation light from a LYSO crystal (light yield of 32000 photons/MeV with a decay time of $40 \mathrm{~ns}$ [9]) to cause a burst of pixels and TDC activations, while the dark count accumulation is much slower.h Therefore an energy equivalent threshold can be set on the number of activated TDCs to distinguish a scintillating event from accumulated dark counts in a frame.

When using the number of occupied TDCs as threshold, it is necessary that the pixel's sensitive time longer than TDCs' sensitive time, so that the chip has a uniform energy response
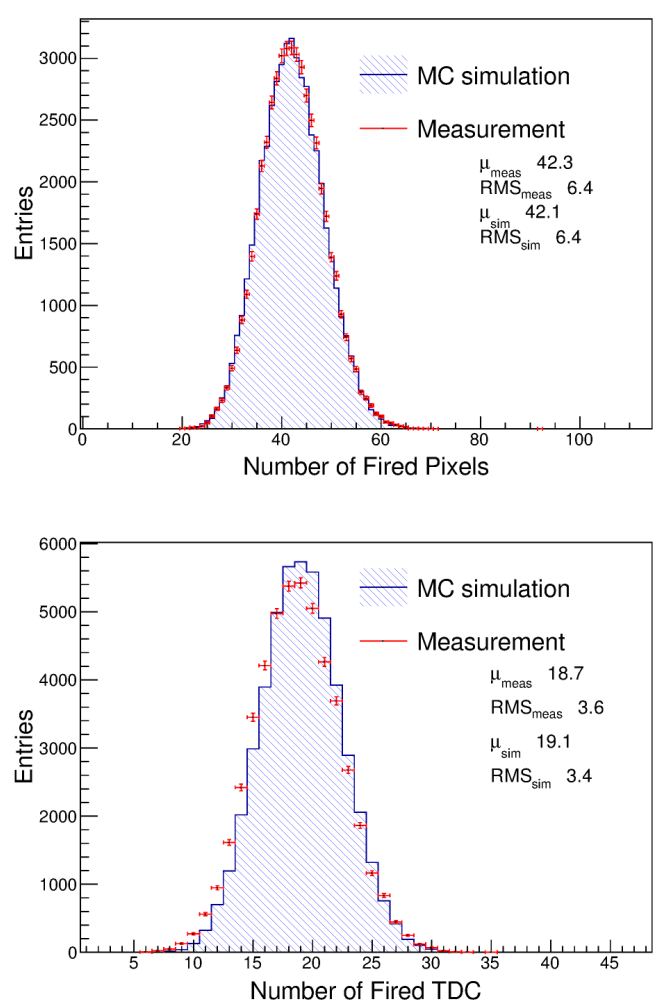

Fig. 5. Comparison between 50,000 simulated frames without incident photon and a measurement taken by a MD-SiPM in the dark. The upper plot shows the comparison between number of fired pixels, the lower plot shows the comparison between number of triggered TDCs.

to a scintillating event which happens during the later part of a frame (cf. Fig. 3).

Fig. 4 shows the functionality test for the SR function. The top plot shows dark counts for different frame acquisition times measured with and without SR function. The SR check interval is set to $200 \mathrm{~ns}$ in the measurement. Without SR function, 160 out of 416 pixels are activated by dark count for a $6 \mu \mathrm{s}$ frame acquisition time. While the SR function keeps the total dark count independent from the length of frame acquisition time. In the bottom plot, the MD-SiPM is used to read out scintillation light from a LYSO crystal irradiated by $511 \mathrm{keV}$ gamma photon from a ${ }^{22} \mathrm{Na}$ source (details of the measurement see section II-E). After applying the SR function with a threshold of 30 TDCs, frames with a valid scintillation event are effectively selected and the multiplicity of noise events is significantly reduced.

\section{MD-SiPM Response Function}

Due to the limited number of pixels of the sensors, the number of recorded photons is not proportional to the number of incident photons. Additionally, with the self-triggered frame readout scheme, a pedestal of dark count further reduces the dynamic range of the sensor. Thus for a linear energy response, it is necessary to correct the detector non-linearity.

A Monte-Carlo approach is chosen for the correction of the detected photons by simulating the detector's response to 


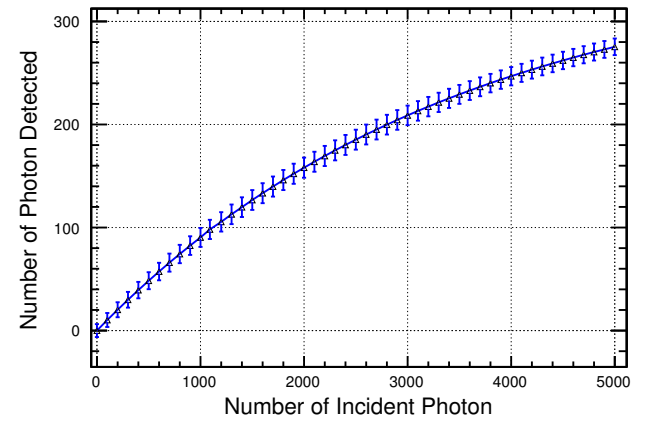

Fig. 6. The MC simulation and digitization tool predicts the detector response to different number of incident photons fall on detector surface. Simulation result points are connected by a line to guide the eye.

different number of incident photons and producing a detector response curve.

The number of fired pixels in response to a given number of incident photons in a given frame acquisition time can be predicted. The developed Monte-Carlo simulation takes all known characteristics of the prototype MD-SiPM into account, including the pixel-by-pixel DCR, pixel masking, pixel to TDC connection scheme and the self-triggered readout logic. Fig 5 shows the comparison of simulated empty frames with the measurement taken by a MD-SiPM in the dark. The slight discrepancy between simulated data and measurements on the activated TDC counts is due to the assumed uniformly distributed dark counts in the simulation while in reality noisy pixels are not uniformly distributed, therefore the probability of triggering each of the 48 TDCs differs. However the nonuniformity of TDC activation probability has no influence on energy response correction.

Fig 6 shows the simulated response curve of the MD-SiPM using $800 \mathrm{~ns}$ frame acquisition time. The detector PDE at $2.5 \mathrm{~V}$ excess bias is $12.5 \%$ [5] and total DCR is 50 Mcps. The simulated incident photons are uniformly distributed over the detector area. A probability density funcion is used to described the arrival time of the photons:

$$
f(t)=\frac{\exp \left(-\frac{t}{\tau_{d}}\right)-\exp \left(-\frac{t}{\tau_{r}}\right)}{\tau_{d}-\tau_{r}}
$$

where $\tau_{r}$ and $\tau_{d}$ is the rise time and decay time of the photon flux. For a LYSO crystal, $100 \mathrm{ps}$ and $40 \mathrm{~ns}$ are used for the rise and decay time respectively. A pedestal of 42 dark counts in the $800 \mathrm{~ns}$ frame acquisition time which is used for scintillator measurement is subtracted from the curve.

\section{E. Scintillator Measurements}

The MD-SiPM is coupled to a $1 \times 1 \times 15 \mathrm{~mm}^{3}$ LYSO crystal produced by Hilger [9] to measure the photon energy spectrum of a ${ }^{22} \mathrm{Na}$ and ${ }^{137} \mathrm{Cs}$ source. Due to the geometry mismatch between the crystal and the MD-SiPM, only $62.4 \%$ of the crystal's surface area is covered by the MD-SiPM as shown in Fig. 7. The crystal is held by a plastic holder and is in dry contact with the sensor. There is no wrapping or coating treatment to the crystal. A frame acquisition time of $800 \mathrm{~ns}$
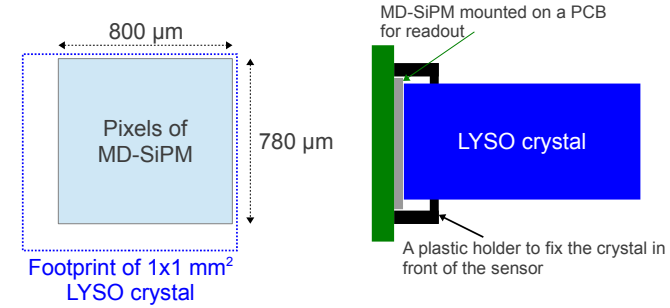

Fig. 7. The $1 \times 1 \times 15 \mathrm{~mm}^{3}$ LYSO crystal in held by a plastic holder and is in dry contact with the MD-SiPM. There is a geometrical mismatch between the size of the crystal and the MD-SiPM sensor size.

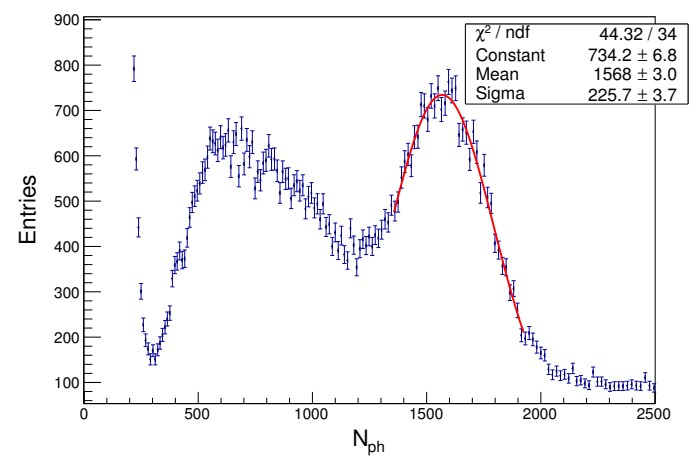

Fig. 8. Light yield spectrum obtain by MD-SiPM coupled with a $1 \times 1 \times$ $15 \mathrm{~mm}^{3}$ LYSO in the unit of incident photons fall on the surface of the detector. The spectrum is corrected for the non-linear response using the MCsimulation curve described in section II-D.

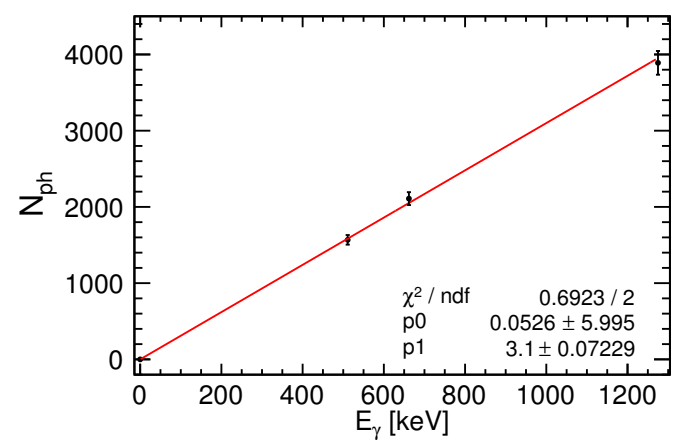

Fig. 9. The system's response to the photo-peak after non-linear correction for the gamma emission of ${ }^{22} \mathrm{Na}(511 \mathrm{keV}$ and $1275 \mathrm{keV})$ and ${ }^{137} \mathrm{Cs}(661.7 \mathrm{keV})$

is used in the measurement. The number of fired pixels and activated TDCs together with their recorded time stamps in each frame are stored.

Fig. 8 shows a spectrum of ${ }^{22} \mathrm{Na}$ source after correcting for the non-linearity. The energy resolution at Full-With-HalfMaximum (FWHM) for the $511 \mathrm{keV}$ photo-peak is $33.9 \%$. Fig. 9 shows the photo-peak position of $511 \mathrm{keV}, 1275 \mathrm{keV}$ from ${ }^{22} \mathrm{Na}$ and $661.7 \mathrm{keV}$ from ${ }^{137} \mathrm{Cs}$ after the non-linearity correction is applied to the spectra. We think the poor energy resolution is a result of the low light yield which is caused by lack of wrapping treatment on the crystal and dry contact between the crystal and the MD-SiPM as well as the geometry mismatch between the crystal and the MD-SiPM. 


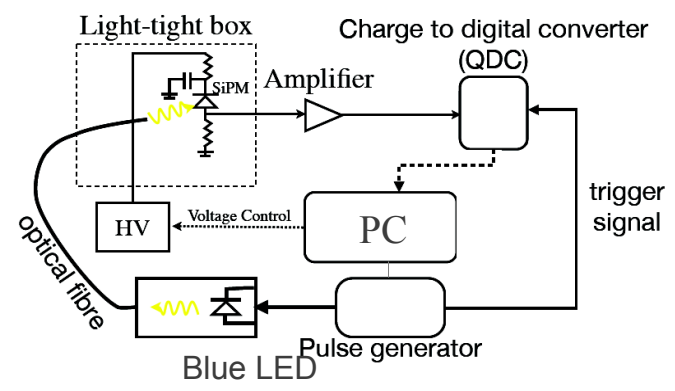

Fig. 10. A diagram shows the setup used to characterize the MPPC. The LED light is turned off during DCR measurement.

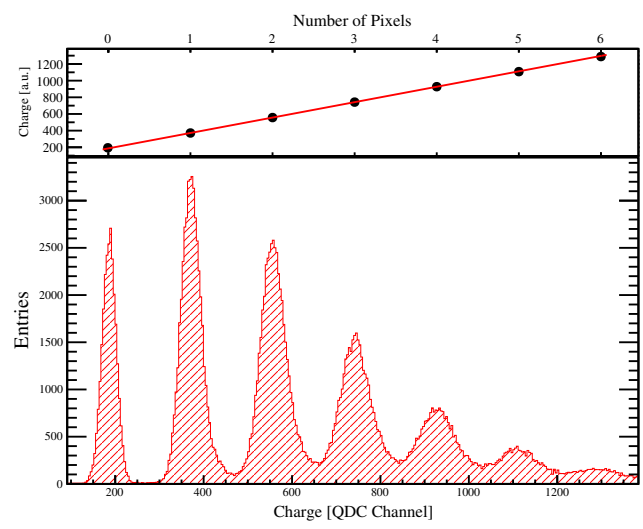

Fig. 11. The histogram of the signal output from MPPC in QDC units. Each peak corresponds to a certain number of pixels fired. By applying a linear fit to the peak positions versus their correspondent pixel chardinality, the pixel's gain of the MPPC can be determined.

This all can be improved for the final measurements but was not possible in this case due to the lack of dedicated crystal for the MD-SiPM and the glueing procedure to attach a bigger crystal to the chip surrounded by fragile wire bond pads.

\section{MPPC SENSOR}

\section{A. Characterization Measurement}

The characterization of MPPC includes the determination of the break down voltage, pixel's charge gain, pixel gain's dependence on the excess bias voltage and the DCR. The Method described in [10] is used. Fig. 10 shows the schematics of the setup used in the characterization measurement. The MPPC is illuminated by a pulsed LED with a wavelength of $405 \mathrm{~nm}$ and a pulse width of about $3 \mathrm{~ns}$. The light intensity is chosen so that on average 2 pixels fire per pulse. The output signal from the MPPC is amplified by a factor of 50 using a Phillips Scientific wide band amplifier (Model 6954) and recorded by a CAEN charge-to-digital converter (QDC965A). The charge integration gate width used is $100 \mathrm{~ns}$. Fig. 11 shows a charge output histogram from the MPPC in QDC unit and the determination of the gain which corresponds to the distance between the peaks in the histogram. The MPPC is operated

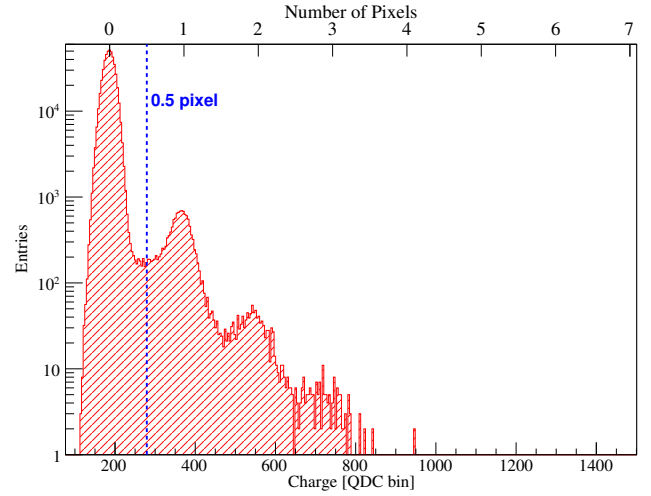

Fig. 12. DCR measurement of the MPPC operated at $1.4 \mathrm{~V}$ excess bias at $20^{\circ} \mathrm{C}$. The dash line shows the level of 0.5 times the gain.

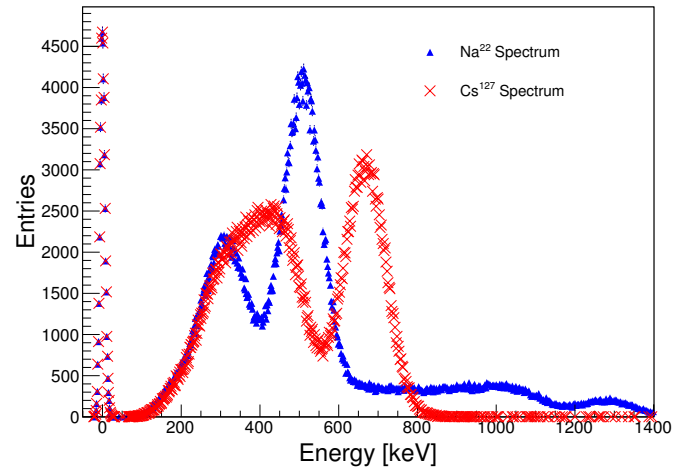

Fig. 13. $\mathrm{Na}^{22}$ and $\mathrm{Cs}^{137}$ gamma emission spectra measured by MPPC coupled to LYSO crystal. The spectra are corrected for the non-linear response of the detector.

at $1.4 \mathrm{~V}$ above the break down voltage. The measurement is performed at $20^{\circ} \mathrm{C}$.

The same setup with LED switched off is used to measure the DCR of the MPPC. Eq. 1 in section II-B is used to calculate the DCR of the MPPC where $P$ is the ratio between the number of events with charge larger than 0.5 times the gain and the total number of events. The charge integration gate used in DCR measurement is $100 \mathrm{~ns}$. Fig. 12 shows the histogram of MPPC's output charge for the DCR measurement. The MPPC is operated at $1.4 \mathrm{~V}$ excess bias and the measurement is performed at $20^{\circ} \mathrm{C}$. The dependence of the MPPC's DCR on the excess bias is shown in the bottom plot of Fig. 1.

\section{B. Scintillator Measurements}

The $1 \times 1 \times 15 \mathrm{~mm}^{3}$ LYSO crystal described in section II-E is coupled to the MPPC. The crystal is used without wrapping or coating treatment and is in dry contact with the MPPC. Energy spectra of ${ }^{22} \mathrm{Na}$ and ${ }^{137} \mathrm{Cs}$ source are measured. The MPPC is operated at $1.4 \mathrm{~V}$ excess bias at $20^{\circ} \mathrm{C}$. The output signal from MPPC is duplicated by a Lecroy $428 \mathrm{~F}$ linear FANIN/FAN-OUT model. One of the two outputs is fed to a low threshold discriminator from CAEN $(\bmod N 96)$ to create a gate of $600 \mathrm{~ns}$ for the QDC. The other output from the FANIN/FAN-OUT model is delayed by about $30 \mathrm{~ns}$ using KX15 coaxial cable and then integrated by the QDC. 
TABLE II

NUMBERS IMPLEMENTED IN THE MPPC SIMULATION

\begin{tabular}{|c|c|}
\hline PDE & $23 \%$ \\
\hline DCR & $500 \mathrm{kcps}$ \\
\hline cross talk & $10.5 \%$ \\
\hline after pulse & $17.7 \%$ \\
\hline recovery time & $9 \mathrm{~ns}$ \\
\hline
\end{tabular}

TABLE III

ENERGY RESOLUTION OF MPPC AND MD-SIPM AT FWHM $\left(\sigma_{E}^{\mathrm{FWHM}} / E\right)$

\begin{tabular}{|c|c|c|}
\hline $\mathrm{E}[\mathrm{keV}]$ & MPPC & MD-SiPM \\
\hline 511 & $23.2 \%$ & $33.9 \%$ \\
\hline 661.7 & $18.8 \%$ & $28.6 \%$ \\
\hline 1275 & $14.7 \%$ & - \\
\hline
\end{tabular}

The simulation program used to simulate MD-SiPM is modified to simulate the MPPC's response. The $600 \mathrm{~ns}$ charge integration time is used as the frame acquisition time. MPPC's pixel cross talk is taken into account by randomly firing one of the 4 direct neighbor pixels with a certain probability if a pixel was fired by a photon. Pixel's recovery time and it's re-firing within the charge integration time is also taken into account. According to [8], a pixel has a $5 \mathrm{~ns}$ dead time after its firing. Its gain then recovers following the exponential function with a time constant of $\tau=9 \mathrm{~ns}$ :

$$
G(t)=1-e^{-(t-5) / \tau}
$$

where $G(t)$ is the ratio of the pixel's gain at time $t$ in nanosecond after the firing to the fully recovered gain. After pulses of the MPPC are taken into account in the simulation. After a pixel fired by a photon, we introduce an additional firing of the same pixel which is uniformly random distributed in time after the initial firing. The ratio between the additionally fired pixels due to after pulse and the total number of pixels fired is the after pulse experimentally measured. For the simulation, we have used cross talk, after pulse and PDE measured in [10]. Table II

Fig. 13 shows the energy spectra for the two sources after non-linear detector response correction. The energy resolution of the photo peaks at Full-Width-Half-Maximum (FWHM) are shown in table III.

\section{COMPARISON OF MD-SIPM AND MPPC}

The operating voltage for the MPPC sample we have used is $71.14 \mathrm{~V}$. According to our characterization, this is $1.4 \mathrm{~V}$ above the break down voltage, and pixel's gain is 7.48e5. The MDSiPM prototype is operated at $22.5 \mathrm{~V}$ which is $2.5 \mathrm{~V}$ above the break down voltage.

The MPPC has a DCR of $0.5 \mathrm{cps} / \mu \mathrm{m}^{2}$ (450 kcps in total) at its nominal operating voltage while the MD-SiPM has $50 \mathrm{cps} / \mu \mathrm{m}^{2}$ (50 Mcps in total). Both devices are operated at $20^{\circ} \mathrm{C}$. In our gamma spectroscopy measurement, given the same signal integration time window, the number of pixels fired by noise for the MPPC is negligible. While in the case of the MD-SiPM, on average 42 dark counts per $6 \mu$ s are accumulated if a "Smart Reset" validation of $800 \mathrm{~ns}$ is used. This reduces the dynamic range of the MD-SiPM from 416 to about 370 pixels.

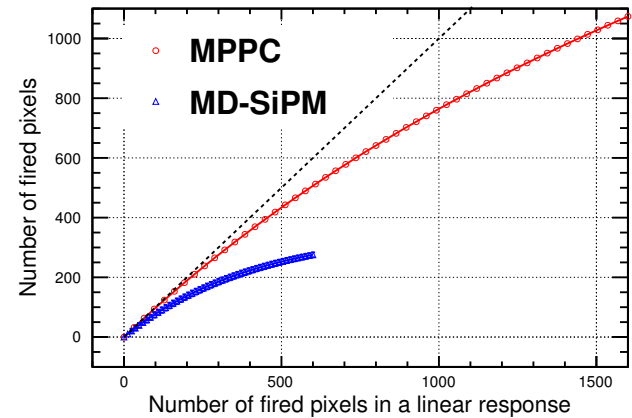

Fig. 14. MC simulation predicted detector response curve of MPPC and the MD-SiPM. The dash line shows the assumed linear response from the sensors respectively.

TABLE IV

DETECTOR RESPONSE TO $511 \mathrm{KEV} \mathrm{GAMMA}$

\begin{tabular}{|c|c|c|}
\hline & MPPC & MD-SiPM \\
\hline \# of pixel fired & 466 & 131 \\
corresponding linear response & 577 & 196 \\
non-linear effect & $19.2 \%$ & $33.2 \%$ \\
\# of incident photon & 1702 & 1568 \\
\hline
\end{tabular}

Both sensors shows strong non-linear response to the scintillation light from the LYSO crystal. The MD-SiPM has 416 pixels and each pixel can be used only once per acquisition frame. MPPC has 400 pixels and a pixel can re-fire with a recovery time constant of $9 \mathrm{~ns}$. Therefore we expect a stronger non-linearity behavior from the MD-SiPM compared to the MPPC. The MPPC has a PDE of 23\% (excluding crosstalk and after pulse) which is almost twice the $12.5 \%$ PDE of the MD-SiPM. Fig. 14 shows the deviation from a ideal linear detector for the MPPC and MD-SiPM respectively. The result is from the MC simulation program. Table IV summarizes the detector response to $511 \mathrm{keV}$ of the two systems. The photons non-linear effect is defined as the percentage decrease of fired pixels to an ideal linear response.

The energy resolution for $511 \mathrm{keV}$ photons at FWHM is $23.2 \%$ for the MPPC and $33.9 \%$ for the MD-SiPM. Both numbers are quoted after applying the non-linear correction. We think the low light yield we have observed in both cases, which is due to the lack of wrapping treatment and the dry contact between the detector and crystal is mainly responsible for the poor energy resolution. The MD-SiPM is further affected by its stronger non-linear behavior due to its pixel's 1-bit memory.

Using the MC simulation program combined with GEANT4 simulated scintillating light from a wrapped LYSO crystal with matched size to the MD-SiPM, we obtain 24\% energy resolution at FWHM for $511 \mathrm{keV}$ gamma radiation for the MD-SiPM. Confirming that the worse result obtained for the digital system is due to a loss of light yield.

\section{CONCLUSION}

We have presented the characterization and scintillator measurement of the prototype MD-SiPM. The concept of using the number of activated TDCs as a threshold instead of using an 
energy threshold to select valid events is proved to be effective. The energy resolution for $511 \mathrm{keV}$ gamma radiation at FWHM of the MD-SiPM is $33.9 \%$. The mismatch between the crystal and the photo-sensor and lack of wrapping treatment to the crystal as well as the low PDE of the chip are the cause of the degradation in energy resolution. A full size MD-SiPM with improved performance for the EndoTOFPET-US project is available for testing soon. And with a dedicated LYSO crystal for the MD-SiPM glued to the sensor to increase the light yield, we expect a better energy resolution from the MDSiPM for the EndoTOFPET-US project. The small geometrical acceptance of the probe detector extention [6] implies the MD$\mathrm{SiPM}$ solution is an essential requirement from the project.

\section{ACKNOWLEDGMENT}

The authors would like to thank Prof. R. Klanner for fruitful discussions. The research leading to these results has received funding from the European Union Seventh Framework Programme (FP7/ 2007-2013) under Grant Agreement no. 256984 (Endo-TOFPET-US).

\section{REFERENCES}

[1] D. Renker, "Geiger-mode avalanche photodiodes, history, properties and problems," Nuclear Instruments and Methods in Physics Research Section A: Accelerators, Spectrometers, Detectors and Associated Equipment, vol. 567, no. 1, pp. 48 56, 2006, proceedings of the 4th International Conference on New Developments in Photodetection. [Online]. Available: http://www.sciencedirect.com/science/article/pii/S0168900206008680

[2] F. Powolny, E. Auffray, S. E. Brunner, E. Garutti, M. Goettlich, H. Hillemanns, P. Jarron, P. Lecoq, T. Meyer, H. Schultz-Coulon, W. Shen, and M. C. S. Williams, "Time-based readout of a silicon photomultiplier (SiPM) for time of flight positron emission tomography (TOF-PET)," Nuclear Science, IEEE Transactions on, vol. 58, no. 3, pp. 597-604, 2011.

[3] I. Britvitch, I. Johnson, D. Renker, A. Stoykov, and E. Lorenz, "Characterisation of geiger-mode avalanche photodiodes for medical imaging applications," Nuclear Instruments and Methods in Physics Research Section A: Accelerators, Spectrometers, Detectors and Associated Equipment, vol. 571, no. 12, pp. 308 - 311, 2007, proceedings of the 1st International Conference on Molecular Imaging Technology, EuroMedIm 2006. [Online]. Available: http: //www.sciencedirect.com/science/article/pii/S0168900206018869

[4] T. Frach, G. Prescher, C. Degenhardt, R. de Gruyter, A. Schmitz, and R. Ballizany, "The digital silicon photomultiplier - principle of operation and intrinsic detector performance," in Nuclear Science Symposium Conference Record (NSS/MIC), 2009 IEEE, 2009, pp. 1959-1965.

[5] S. Mandai, V. Jain, and E. Charbon, "A fully-integrated $780 \times 800 \mu \mathrm{m}^{2}$ multi-digital silicon photomultiplier with column-parallel time-to-digital converter," in ESSCIRC (ESSCIRC), 2012 Proceedings of the, 2012, pp. 89-92.

[6] "Endo-TOFPET-US Proposal: "Novel multimodal endoscopic probes for simultaneous PET/ultrasound imaging for image-guided interventions"," 12010 , european Union 7th 186 Framework 187 Program (FP7/20072013) under Grant Agreement No. 256984.

[7] S. Mandai and E. Charbon, "A $4 \times 4 \times 416$ digital SiPM array with 192 TDCs for multiple high-resolution timestamp acquisition," Journal of Instrumentation, vol. 8, no. 05, pp. 5-24.

[8] MPPC S10362-11-050P datasheet. [Online]. Available: http://www. hamamatsu.com

[9] Hilger LYSO(Ce) crystal. [Online]. Available: http://www. hilger-crystals.co.uk/properties.asp?material $=18$

[10] P. Eckert, H.-C. Schultz-Coulon, W. Shen, R. Stamen, and A. Tadday, "Characterisation studies of silicon photomultipliers," Nuclear Instruments and Methods in Physics Research Section A, vol. 620, pp. 217 226, 2010. 